\title{
Flow bifurcation and heat transfer in a spherical gap
}

\author{
W.-J. Luo, R.-J. Yang* \\ Department of Engineering Science, National Cheng Kung University, Tainan 70101, Taiwan
}

Received 9 February 1999; received in revised form 24 May 1999

\begin{abstract}
This paper investigates the steady flow and heat transfer between two concentric spheres with the inner one rotating and the outer one stationary for the gap width of $\sigma=0.18$. A continuation method is applied to study the bifurcation structure of the discretized governing equations. The resulting Jacobian matrix along with a test function approach is used to locate the bifurcation points. A bifurcation diagram of the flow is constructed for $R e<1200$. Seven steady flow modes are predicted. Linear stability analysis is used to determine the stability of each flow mode. Temperature distribution and heat transfer rate of each flow mode are also computed and analyzed. (C) 2000 Elsevier Science Ltd. All rights reserved.
\end{abstract}

\section{Introduction}

Spherical Taylor-Couette flow has been an important research topic for many years, and has been studied by many researchers. Wimmer [1] showed in his experiments that the flow modes could be produced by different acceleration histories of the inner sphere. Bartels [2] solved the unsteady axisymmetric NavierStokes equations in stream-function vorticity formulation by means of a finite-difference approximation. He used a quadrant annulus $(0 \leq \theta \leq \pi / 2)$ as a computational domain and imposed equatorially symmetric boundary conditions. He could not obtain 1-vortex flow without arbitrary disturbances near the equator. Marcus and Tuckerman [3] used the same formulation with the equations solved by a pseudospectral method for full domain $(0 \leq \theta \leq \pi)$. Three steady flow modes with $0-, 1$ - and 2- Taylor vortices are found to be symmetric with respect to the equator. They also examined

\footnotetext{
* Corresponding author. Tel.: + 886-6-275-8506; fax: + 8866-276-6549.

E-mail address: rjyang@mail.ncku.edu.tw (R.J. Yang).
}

the transitions of the three flow modes. Schrauf [4] used the continuation method of Keller [5] to investigate how the stability of spherical Couette flow depends on the gap size. Bühler [6] presented a comprehensive investigation of a spherical gap flow, $\sigma=0.154$, with an initial value code based on an explicit finite difference method. He discovered for the first time that, within the non-existence range of the symmetric 1-vortex mode, a similar 1-vortex stable mode asymmetric with respect to the equatorial plane could exist. Bar-Yoseph et al. [7] considered both concentric and eccentric spherical gaps for two different radii ratios of a medium size gap by means of a finiteelement method. Mamun and Tuckerman [8] adopted a pseudospectral time-stepping formulation to study the bifurcation-theoretic genesis of Bühler's asymmetric states. They found that the asymmetric branch originates from pitchfork bifurcation; its stabilization occurs via a subsequent subcritical Hopf bifurcation. Yang [9] used the finite-difference method with fictitious symmetric boundary conditions to find all possible stable steady axisymmetric flow modes. Both symmetric and asymmetric solutions can be predicted in a deterministic way. 


\author{
Nomenclature \\ $C_{\mathrm{p}} \quad$ specific heat at constant pressure \\ $e_{l} \quad l$-th unit vector \\ $k \quad$ thermal conductivity of the fluid $\left[\mathrm{W} \mathrm{m}^{-1} \mathrm{~K}^{-1}\right.$ ] \\ $N u_{\mathrm{i}}$ local Nusselt number at inner sphere surface, \\ defined by Eq. (24) \\ $\bar{N} u_{\mathrm{i}} \quad$ average Nusselt number at inner sphere sur- \\ face, defined by Eq. (25) \\ $N u_{\mathrm{o}}$ local Nusselt number at outer sphere surface, \\ defined by Eq. (24) \\ $\bar{N} u_{\mathrm{o}}$ average Nusselt number at outer sphere sur- \\ face, defined by Eq. (25) \\ $\operatorname{Pr} \quad$ Prandtl number, $\rho C_{\mathrm{p}} v / k$ \\ $r$ radial coordinate \\ $r^{*} \quad$ dimensionless radial coordinate, $r / R_{\mathrm{i}}$ \\ Re Reynolds number, $\omega R_{\mathrm{i}}^{2} / v$ \\ $R_{\mathrm{i}} \quad$ radius of inner sphere \\ $R_{\mathrm{O}} \quad$ radius of outer sphere \\ $s$ arc length \\ $T$ temperature \\ $T_{\mathrm{i}} \quad$ temperature in the inner sphere \\ $T_{\mathrm{o}}$ temperature in the outer sphere \\ $u^{*} \quad$ non-dimensional velocity \\ $u^{\mathrm{r}} \quad$ velocity in the radial coordinate \\ $u^{\theta} \quad$ velocity in the meridional coordinate \\ $u^{\phi} \quad$ velocity in the azimuthal coordinate \\ $X \quad$ solution vector, $\left[\left(\psi_{1}, \xi_{1}, \phi_{1}\right), \ldots\left(\psi_{\mathrm{n}}, \xi_{\mathrm{n}}, \phi_{\mathrm{n}}\right)\right]$
}

\section{Greek symbols}

$\theta \quad$ meridional coordinate

$\sigma$ gap size ratio, normalized by the inner sphere radius

$\beta \quad$ shift parameter in the Arnoldi method

$\gamma \quad$ eigenvalue of the defined system

$\varepsilon \quad$ a small disturbance vector

$\omega$ angular velocity

$\psi$ stream function

$\xi \quad$ vorticity function

$\phi$ angular velocity function

$\psi^{*}$ non-dimensional stream function

$\xi^{*}$ non-dimensional vorticity function

$\phi^{*}$ non-dimensional angular velocity function

$\alpha \quad$ ratio of outer and inner radius, $R_{\mathrm{o}} / R_{\mathrm{i}}$

$\lambda$ continuation parameter

$\delta \lambda$ small increment in the continuation parameter

$\Gamma_{\mathrm{i}, \mathrm{j}}$ test function

$\Theta$ non-dimensional temperature, $\left(T-T_{\mathrm{o}}\right) /\left(T_{\mathrm{i}}-T_{\mathrm{o}}\right)$

$\rho$ fluid density

$\checkmark$ kinematic viscosity
Subscripts
i inner sphere surface
o outer sphere surface

Heat transfer between concentric spheres has also been studied by many researchers. They concerned mainly natural convection between concentric spheres, whose inner and outer parts are both stationary. Experimental research on natural convection in annuli between two isothermal concentric spheres has been described by Bishop et al. [10,11], Scanlan et al. [12] and Yin et al. [13] for a very wide range of Prandtl and Rayleigh numbers. Temperature distribution within the annuli was measured and Nusselt-Grashof number correlations were presented. Burns and Tien [14] used both finite-difference and regular perturbation method for the study of natural convection in porous media bounded by concentric spheres and horizontal cylinders. Singh and Elliott [15] carried out a power series solution for a thermally stratified medium between concentric spheres. The inner sphere was kept either at a constant temperature or constant heat flux, and the outer sphere was maintained at a variable temperature for vertical stratification. Nelsen and Douglass [16,17], and Wright and Douglass [18] analyzed natural convection in a spherical annuli filled with a heat-generating fluid. These studies concentrated on a single flow mode resulting from natural heat convection without considering multiple solutions.

In this study, a continuation method proposed by Keller [5] is applied to the Navier-Stokes equations in order to find possible solution branches for a spherical Taylor-Couette flow. A test function, which was proposed by Seydel [19], is applied to locate the singular points. It can reduce computing costs and avoid the scaling problems in numerics. Moreover, for the heat transfer between concentric spheres, past studies have concentrated on the natural convection flow in a stationary spherical annulus with isothermal walls. However, for the inner rotating and outer stationary concentric annulus with a small temperature difference, the flow is driven mainly by the drag of the inner sphere. Therefore, in the flow, the natural convection effect is much weaker than the viscous effect, and can be neglected in the present study. It would be interesting to investigate the heat transfer associated with the multiple flow modes. In this study, a bifurcation diagram is constructed and compared with those in the literature. Finally, the predicted solutions in terms of temperature distribution and heat transfer between the spheres for different solution modes are analyzed. 


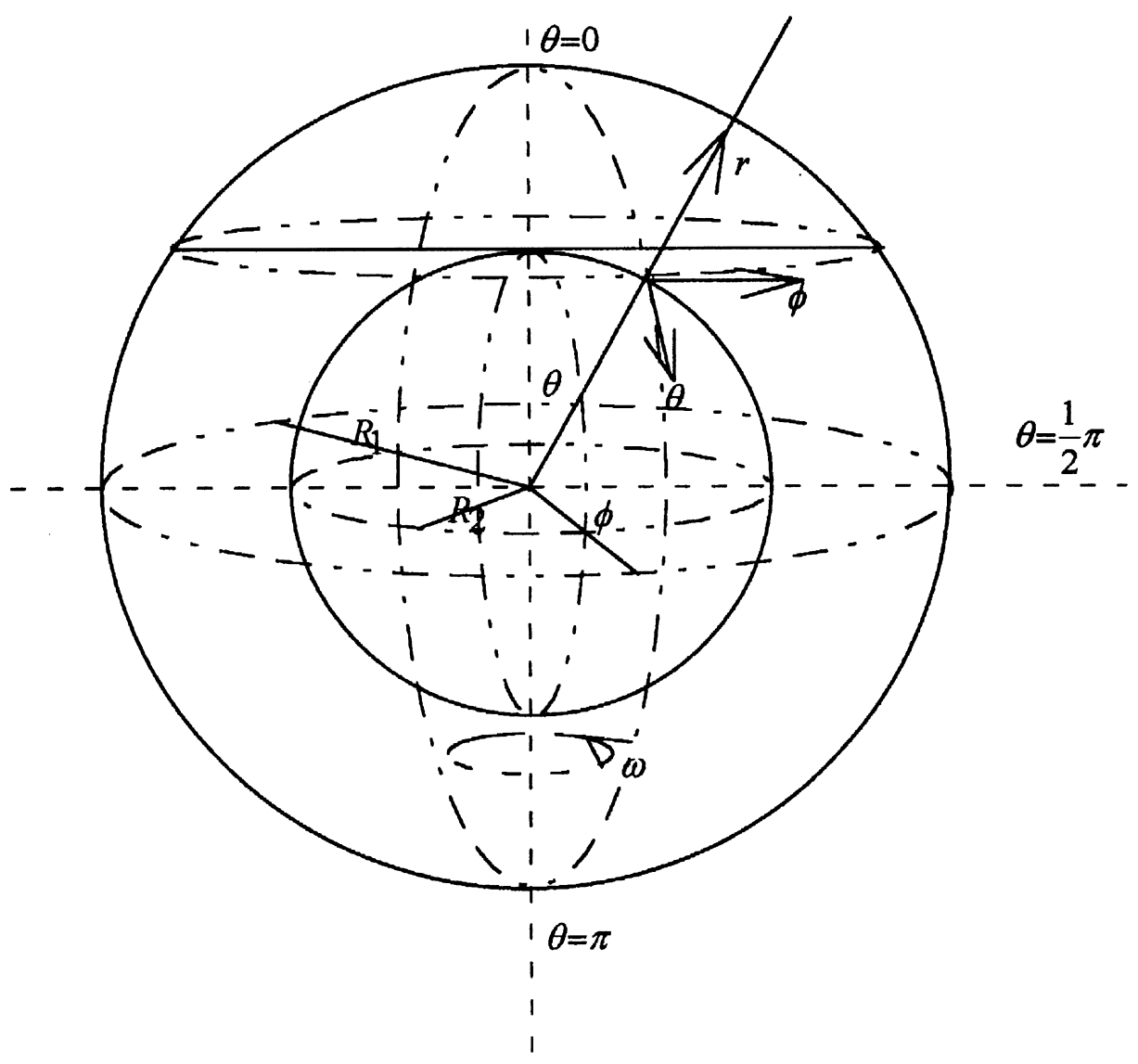

Fig. 1. The coordinate system of the spherical annulus.

\section{Governing equations and numerical methods}

We consider a flow of steady, incompressible Newtonian fluid contained between two concentric spheres. The outer sphere is stationary while the inner one rotates. The inner sphere rotates with angular velocity $\omega$, as shown in Fig. 1. Since axisymmetric flows are considered, stream function $\psi$, vorticity function $\xi$, and angular velocity function $\phi$ can be introduced as follows:

$u^{\mathrm{r}}(r, \theta)=\frac{1}{r \sin \theta} \frac{1}{r} \frac{\partial}{\partial \theta} \psi(r, \theta)$

$u^{\theta}(r, \theta)=-\frac{1}{r \sin \theta} \frac{\partial}{\partial r} \psi(r, \theta)$

$u^{\phi}(r, \theta)=\frac{1}{r \sin \theta} \phi(r, \theta)$ $(\nabla \times u)^{\phi}=\frac{1}{r}\left[\frac{\partial}{\partial r}\left(r u^{\theta}\right)-\frac{\partial}{\partial \theta} u^{\mathrm{r}}\right]=\frac{1}{r \sin \theta} \xi(r, \theta)$.

Let the radius of the inner sphere $R_{\mathrm{i}}$ and angular velocity $\omega$ be reference values of radius and angular velocity, respectively. Then non-dimensional quantities (denoted by asterisks) can be defined via

$$
\begin{gathered}
r=r^{*} R_{\mathrm{i}}, \quad u=u^{*} R_{\mathrm{i}} \omega, \quad \psi=\psi^{*} R_{\mathrm{i}}^{3} \omega, \\
\phi=\phi^{*} R_{\mathrm{i}}^{2} \omega, \quad \xi=\xi^{*} R_{\mathrm{i}} \omega .
\end{gathered}
$$

We drop the asterisks in the following sections, and the Reynolds number can be defined as

$$
R e=\omega R_{\mathrm{i}}^{2} / v
$$

The dimensionless momentum equation in the circumferential direction is 


$$
\begin{aligned}
& \frac{\partial \psi}{\partial \theta} \frac{\partial \phi}{\partial r}-\frac{\partial \psi}{\partial r} \frac{\partial \phi}{\partial \theta}=\frac{r^{2} \sin \theta}{R e} D^{2} \phi \\
& 2 \xi\left[\cot \theta \frac{\partial \psi}{\partial r}-\frac{1}{r} \frac{\partial \psi}{\partial \theta}\right]-2 \phi\left[\cot \theta \frac{\partial \phi}{\partial r}\right. \\
& \left.\quad-\frac{1}{r} \frac{\partial \phi}{\partial \theta}\right]-\left[\frac{\partial \psi}{\partial r} \frac{\partial \xi}{\partial \theta}-\frac{\partial \psi}{\partial \theta} \frac{\partial \xi}{\partial r}\right]=\frac{r^{2} \sin \theta}{R e} D^{2} \xi
\end{aligned}
$$

and the streamfunction follows from the Poisson equation

$-D^{2} \psi-\xi=0$

where the differential operator is

$D^{2}=\frac{\partial^{2}}{\partial r^{2}}+\frac{1}{r^{2}} \frac{\partial^{2}}{\partial \theta^{2}}-\frac{\cot \theta}{r^{2}} \frac{\partial}{\partial \theta}$.

$\alpha$ is defined as $\alpha=R_{\mathrm{o}} / R_{\mathrm{i}}=\sigma+1$.

The boundary conditions are 'no slip' conditions at the spheres

$\phi(1, \theta)=\sin ^{2} \theta, \quad \phi(\alpha, \theta)=0$,

$\psi(1, \theta)=0, \quad \psi(\alpha, \theta)=0$,

$\xi(1, \theta)=-\psi_{\mathrm{rr}}, \quad \xi(\alpha, \theta)=-\psi_{\mathrm{rr}}$,

and the symmetry conditions at the poles are

$\phi(r, 0)=0, \quad \phi(r, \pi)=0$,

$\psi(r, 0)=0, \quad \psi(r, \pi)=0$

$\xi(r, 0)=0, \quad \xi(r, \pi)=0$.

There are no physical conditions for the vorticity function at the walls. Hence, Poisson equations are used

$\xi(1, \theta)+\psi_{\mathrm{rr}}(1, \theta)=0, \quad \xi(\alpha, \theta)+\psi_{\mathrm{rr}}(\alpha, \theta)=0$.

The governing equations are discretized by central differences of second order and form a system of nonlinear algebraic equations

$G(X, \lambda)=0$,

where $X$ is the solution vector, and $\lambda$ stands for the Reynolds number. When $\lambda=0$ a solution of the continuous problem can be obtained by numerical methods. As $\lambda$ deviates from zero, we can use the solution as an initial estimate of the discrete solution in the Euler-Newton method applied to the Eq. (14). This gives a sequence of iterates $\left[X^{(v)}(\lambda)\right]$ defined by

$X^{(0)}(\lambda) \equiv$ initial estimate,

$$
\begin{gathered}
G_{X}\left(X^{(v)}, \lambda\right)\left[X^{(v+1)}-X^{(v)}\right]=-G\left(X^{(v)}, \lambda\right), \\
v=0,1,2, \ldots
\end{gathered}
$$

Here is the Jacobian matrix of Eq. (14). One way to obtain good initial estimates is to use a Taylor expansion of the solution with respect to the changes in the parameter $\lambda$. Thus we use

$X^{(0)}(\lambda+\delta \lambda)=X(\lambda)+\delta \lambda X_{\lambda}(\lambda)$

To obtain $X_{\lambda}$, we can use Eq. (14) and it satisfies

$G_{X}(X, \lambda) X_{\lambda}=-G_{\lambda}(X, \lambda)$.

The method described in Eqs. (15) and (16) is known as the Euler-Newton continuation. It is extremely effective and usually converges quadratically. However, it fails in points where the Jacobian matrix $G_{X}(X$, $\lambda)=0$ is singular. To avoid the singular points, the Keller's [5] continuation method is introduced

$$
\begin{gathered}
N(X(s, \lambda(s))) \equiv<\dot{X}\left(s_{0}\right)\left[X(s)-X\left(s_{0}\right)\right]> \\
\quad+\dot{\lambda}\left(s_{0}\right)\left[\lambda(s)-\lambda\left(s_{0}\right)\right]-\left(s-s_{0}\right)=0
\end{gathered}
$$

Here $\left[X\left(s_{0}, \lambda\left(s_{0}\right)\right)\right]$ is a previously computed solution for $\lambda$ fixed in the present discussion and $s=s_{0} . \dot{X}=\mathrm{d} X /$ $\mathrm{d} s$ and $\dot{\lambda}=\mathrm{d} \lambda / \mathrm{d} s$ denote the components of a tangent vector to the solution path $[X(s), \lambda(s)]$. Then, a new system of equations written as

$\left\{\begin{array}{l}G(X, \lambda)=0 \\ N(X, \lambda, s)=0\end{array}\right.$

can be solved. The Jacobian of this new system is

$\frac{\partial(G, N)}{\partial(X, \lambda)}=\left(\begin{array}{cc}G_{X} & G_{\lambda} \\ N_{X} & N_{\lambda}\end{array}\right)$.

With the Euler-Newton continuation in $s$ rather than $\lambda$, it is possible to follow the solution around singular points.

The singular points can be located at the place where the sign of the determinant of the Jacobian matrix changes. It is necessary to use a lot of computer memory storage and CPU time to calculate these singular points which may suffer from scaling problems in numerics. To reduce computer costs and avoid the scaling problems, we introduce a test function, $\Gamma_{i, j}(X$, $\lambda$ ), proposed by Seydel [19], shown as follows:

$\Gamma_{i, j}(X, \lambda)=e_{l}^{\mathrm{T}} \tilde{J}(X, \lambda) \mathbf{h}$

where $\mathbf{h}$ satisfies the system

$\mathbf{h}=\tilde{J}_{i, j}^{-1} e_{i}$ 
and $e_{i}$ is a unit column vector and the matrix $\tilde{J}_{i, j}$ a reduced $\tilde{J}$ by replacing its $i$-th row by a unit base vector, i.e. $\tilde{J}_{i}, j \equiv\left\lfloor\left(I-e_{i} e_{i}^{\mathrm{T}}\right) \tilde{J}+e_{i} e_{j}^{\mathrm{T}}\right\rfloor$. Eq. (20) guarantees that the column vector $\mathbf{h}$ is a nontrival solution. The value of Eq. (19), $\Gamma_{i, j}(X, \lambda)$, approaches zero if $G_{X}(X$, $\lambda)$ approaches singular points. The quantity $\Gamma_{i, j}(X, \lambda)$ measures the rank-deficiency and serves as a test function. Therefore, the singular points can be determined by monitoring the value of the test function. If the sign of the test function changes, a flow mode variation is encountered. However, $\Gamma_{i, j}(X, \lambda)$ is not singular at simple turning points. Detecting turning points is much easier through the sign change of $\partial \lambda / \partial s$.

Regarding the heat transfer prediction, it is assumed that the inner sphere is kept at a constant temperature $T_{\mathrm{i}}$, while the outer sphere is still maintained at $T_{0}$, with $T_{\mathrm{i}}>T_{0}$. Furthermore, it is assumed that (1) fluid properties are constant, and (2) viscous dissipation and radiation effects can be neglected. The temperature field is described by the energy conservation equation in the spherical coordinates:

$$
\begin{aligned}
& u^{\mathrm{r}} \frac{\partial \Theta}{\partial r}+\frac{u^{\theta}}{r} \frac{\partial \Theta}{\partial \theta}=\frac{1}{\operatorname{RePr}}\left[\frac{\partial^{2} \Theta}{\partial r^{2}}+\frac{2}{r} \frac{\partial \Theta}{\partial r}+\frac{\cot \theta}{r^{2}}\right. \\
& \left.\frac{\partial \Theta}{\partial \theta}+\frac{1}{r^{2}} \frac{\partial^{2} \Theta}{\partial \theta^{2}}\right]
\end{aligned}
$$

where

$\Theta=\frac{T-T_{0}}{T_{\mathrm{i}}-T_{0}}, \quad \operatorname{Pr}=\frac{\rho C_{\mathrm{p}} v}{\kappa}$.

The boundary conditions are given as follows:

$\Theta(1, \theta)=1, \quad \Theta(\alpha, \theta)=0$,

$\frac{\partial \Theta(r, 0)}{\partial \theta}=0, \quad \frac{\partial \Theta(r, \pi)}{\partial \theta}=0$.

In this study, Prandtl number $=0.72$ was used. The local Nusselt number can be obtained from the gradient of the temperature at the inner and outer boundaries from the following:

$$
\begin{aligned}
& N u_{\mathrm{i}}=-\frac{\alpha-1}{\alpha}\left(\frac{\partial \Theta}{\partial r}\right)_{r=1}, \\
& N u_{\mathrm{o}}=-\alpha(\alpha-1)\left(\frac{\partial \Theta}{\partial r}\right)_{r=\sigma} .
\end{aligned}
$$

The average Nusselt numbers are defined as:
$\bar{N} u_{\mathrm{i}, 0}=-\int_{0}^{\pi} N u_{\mathrm{i}, 0}\left[\left(\frac{\sin \theta}{2}\right)\right] \mathrm{d} \theta$.

To investigate the stability of various flow states obtained by the continuation method described above, a linear stability analysis is carried out. A basic state $X_{\mathrm{o}}$ found by Newton's method during continuation is perturbed by small time-dependent quantities,

$X=X_{\mathrm{o}}+\varepsilon \mathrm{e}^{\gamma \tau}$,

where $\varepsilon$ is a small disturbance vector. For transient solutions, a set of time-dependent equations can be derived and written as:

$M(X) \frac{\mathrm{d} X}{\mathrm{~d} \tau}=G(X, \lambda)$

where $M(X)$ is the mass matrix and it is singular because some equations, e.g. the stream equations, do not have an explicit time dependent term. Substituting Eq. (26) into Eq. (27), after collecting the linear terms of $\varepsilon$, leads to the generalized algebraic eigenvalue problem:

$\gamma M\left(X_{\mathrm{o}}\right) \varepsilon=\tilde{J}\left(X_{\mathrm{o}}\right) \varepsilon$.

The matrix $\tilde{J}$ represents the Jacobian matrix of $G(X$, $\lambda$ ) evaluated for the basic state solution. The stability of the basic state $X_{\mathrm{o}}$ can be determined by the sign of the eigenvalue $\gamma$. The basic solution is infinitesimally stable if $\operatorname{Re}\{\gamma\}<0$ holds for all eigenvalues $\gamma$. If there is at least one eigenvalue with $\operatorname{Re}\{\gamma\}>0$, the corresponding eigenmode will grow as $t \rightarrow \infty$ and the basic solution is unstable. However, because $M$ is singular, some eigenvalues are infinite and they are not responsible to the linear instability. It is necessary to remove these infinite eigenvalues when calculating the leading eigenvalues. An effective algorithm for this purpose is a shift-and-inverse operation (Arnoldi algorithm; Saad [20]). The generalized eigenvalue problem Eq. (28) is transformed into a standard eigenvalue problem

$(\tilde{J}-\beta M)^{-1} M \varepsilon=\hat{\gamma} \varepsilon, \quad \hat{\gamma}=\frac{1}{\gamma-\beta}$,

where $\beta$ is a complex shift parameter such that $(\tilde{J}-\beta M)^{-1} M$ is not singular. ARPACK [21], an Arnoldi-method based package, is then used to calculate the leading eigenvalues and corresponding eigenvectors. Real shift values are found satisfactory in the present study. The onset of instability in our calculation, where the leading eigenvalue becomes positive, is consistent with the bifurcation diagram or the test function. 
Table 1

Critical Reynolds numbers for the transition from a flow with $\mathrm{m}$ vortex pairs to a flow with $\mathrm{n}$ vortex pairs; -indicates that the data are not reported

\begin{tabular}{|c|c|c|c|c|c|}
\hline & $\sigma$ & $0 \rightarrow 1$ & $1 \rightarrow 0$ & Hysteresis $(\Delta R e)$ & $0 \rightarrow 2$ \\
\hline \multicolumn{6}{|l|}{ Numerical results } \\
\hline \multicolumn{6}{|l|}{ This study } \\
\hline$(31 \times 301)$ & 0.18 & 650.23 & 643.6 & 6.63 & $745.7 / 790^{\mathrm{c}}$ \\
\hline$(41 \times 301)$ & 0.18 & 649.13 & 642.32 & 6.81 & $745.1 / 789^{\mathrm{c}}$ \\
\hline$(41 \times 361)$ & 0.18 & 649.13 & 642.32 & 6.81 & $744.3 / 788^{\mathrm{c}}$ \\
\hline Marcus and Tuckerman & 0.18 & 652.11 & 644.25 & 7.86 & $739.84 / 775^{\circ}$ \\
\hline Schrauf $[4]$ & 0.18 & 659.97 & - & - & - \\
\hline Schrauf $^{\mathrm{a}}$ & 0.176 & - & 652.89 & - & 750.01 \\
\hline Schrauf ${ }^{b}$ & 0.176 & 666.32 & 660.87 & 5.45 & \\
\hline \multirow[t]{2}{*}{ Bar-Yoseph [7] } & 0.176 & - & 665 & - & - \\
\hline & 0.18 & 666 & - & - & 753 \\
\hline Bartels [2] & 0.176 & - & 666.5 & - & $728-776.5$ \\
\hline \multicolumn{6}{|l|}{ Experimental results } \\
\hline Wimmer [1] & 0.18 & $649.5-653.4$ & $624.6-653$ & - & $800-810.6$ \\
\hline
\end{tabular}

${ }^{a}$ Taken from the values listed in Marcus and Tuckerman [3].

${ }^{\mathrm{b}}$ Taken from the values listed in Bar-Yoseph [7].

${ }^{\mathrm{c}}$ The first value is the Reynolds number that the 2-vortex flow starts to appear and the second value is the Reynolds number where the flow bifurcation is detected.

\section{Results and discussion}

\subsection{Comparison with other calculations and experiments}

In order to check the dependency of the numerical solution on grid point distribution, three different kinds of grid points, $31 \times 301,41 \times 301,41 \times 361$ in radial and meridional direction, respectively, are chosen for the investigation. For $\sigma=0.18$ and $\sigma=0.176$ several numerical and experimental investigations are available in the literature. A comparison of our results with those of Marcus and Tuckerman [3], Schrauf [4], Bar-Yoseph et al. [7], Bartels [2] and Wimmer [1] is listed in Table 1. The present solutions can be regarded as grid-independent solutions when the $41 \times 301$ grid system is used. The computed critical Reynolds numbers agree quite well with other results as shown in Table 1.

\subsection{Solution states}

Fig. 2 depicts the seven different steady flow modes predicted for $\sigma=0.18$ in this study. The flow modes shown in Fig. 2(a)-(d) have been known in the literature, namely as 0 -vortex, 0 -vortex with pinches, 1 -vortex and 2-vortex flow, respectively. The other three flow modes are reported here for the first time. For simplicity, we represent the domain as rectangular, although it is actually curved. Contours of $\psi r \sin \theta$, which are tangent to the meridional velocity, are plotted. The solid curves designate counter-clockwise circulation, and the dashed curves clockwise circu- lation. In these flows, Ekman pumping caused fluid to be thrown outward centrifugally along the rotating inner sphere and pulled from the center of the stationary outer sphere, causing large-scale meridional flow whose direction is counter-clockwise in the northern hemisphere, and clockwise in the southern hemisphere. This large-scale circulation can be seen in all flows with non-zero Reynolds numbers.

Similar to Fig. 2(c), Fig. 2(e) contains one pair of Taylor vortices near the equator. However, its vortex size is much smaller and its strength is much weaker than that in Fig. 2(c). Fig. 2(g) is the asymmetric 1vortex with a pinch predicted at $\mathrm{Re}=1000$, containing two Taylor-vortices, one of which is larger and straddles the equator. Furthermore, this flow mode is characterized by a pinch on only one of the large-scale recirculations. The asymmetric 2 -vortex state shown at $R e=800$ in Fig. 2(f) consists of two pairs of Taylor vortices, one of which is only slightly larger than the other pair and straddles the equator as well.

\subsection{Heat transfer for different solution states}

Fig. 3(a) represents isotherms of the 0-vortex mode at $R e=514$. In this figure, constant-temperature contours were nearly parallel to the spherical boundaries. Therefore, heat was being transferred from the inner sphere to the outer sphere purely by heat conduction, and the effect of flow convection could be ignored. Fig. 4(a) shows the local hemispheric Nusselt number distributions. The solid and dashed lines represent the local Nusselt numbers for the inner and outer spheres 


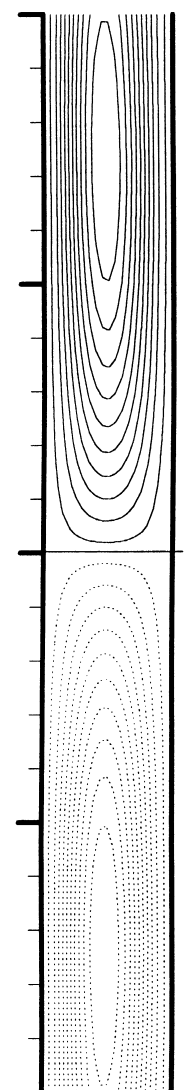

(a) $\mathrm{Re}=600$

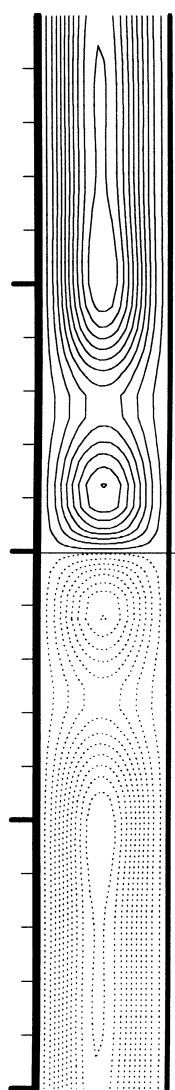

(b) $\mathrm{Re}=700$

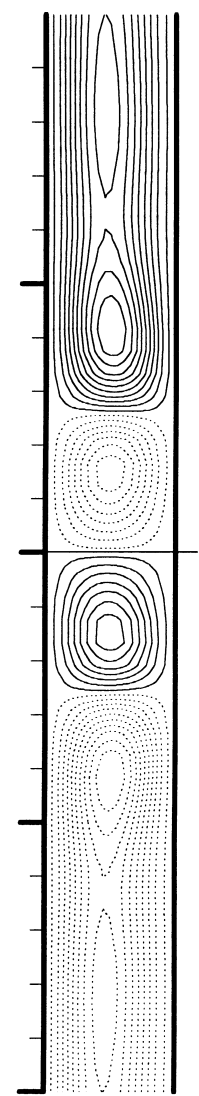

(c) $\mathrm{Re}=1000$

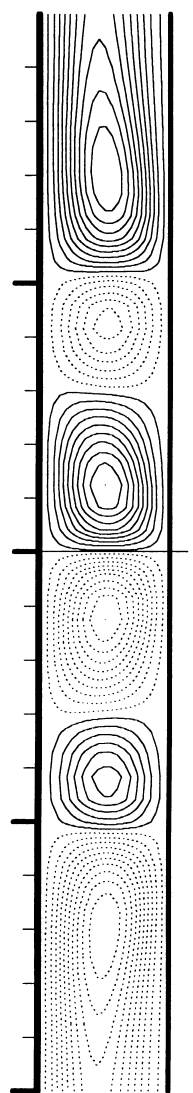

(d) $\mathrm{Re}=1000$

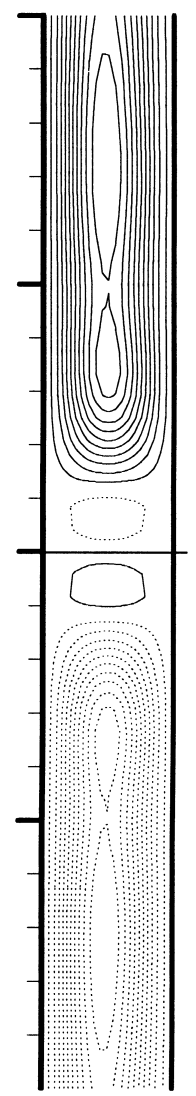

(e) $\operatorname{Re}=1000$

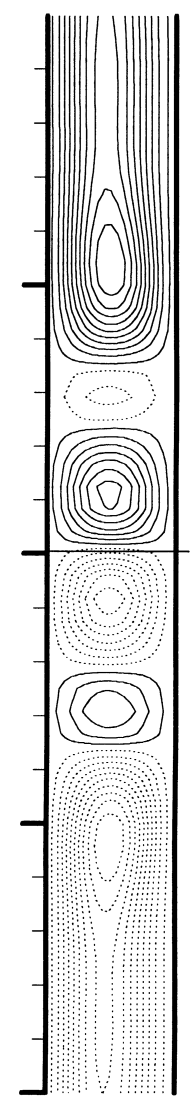

(f) $\operatorname{Re}=800$

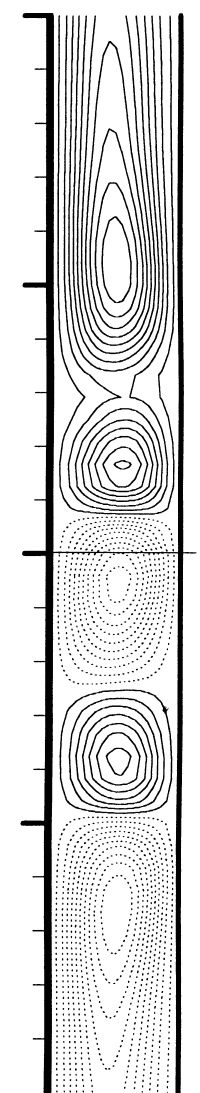

(g) $\mathrm{Re}=1000$

\section{Indicates the position of the equator.}

Fig. 2. Seven different steady flow modes predicted for $\sigma=0.18$ in this study.

$\left(N u_{\mathrm{i}}, N u_{\mathrm{o}}\right)$, respectively, as defined in Eq. (24). At the poles, cold fluid was pulled from the outer sphere to the inner sphere, and heat transfer from the inner sphere was at its greatest. Therefore, there was a local maximum for the $N u_{\mathrm{i}}$ distribution. Afterwards, fluid moving down to the equator along the inner sphere was heated gradually by the hot wall. Meanwhile, the temperature gradient in the radial direction and $N u_{\mathrm{i}}$ decreased gradually forming a local $N u_{\mathrm{i}}$ minimum at the equator. By contrast, at the equator, where the outer sphere received heat from the hot radial outflow, heat transfer at the outer sphere was at its greatest, thereby forming a local maximum of $N u_{\mathrm{o}}$ distribution. Fluid returning to the poles along the outer sphere was then cooled gradually by the cold wall. Meanwhile, the temperature gradient in the radial direction and $N u_{\mathrm{o}}$ decreased gradually forming local minimal of $N u_{\mathrm{o}}$ at the poles. From these results, we can conclude that extremes of $N u_{\mathrm{i}}$ and $N u_{\mathrm{o}}$ always exist at where radial inflow and radial outflow locate. At radial inflow lo- cations, the extremes of $N u_{\mathrm{i}}$ and $N u_{\mathrm{o}}$ are locally maximal and locally minimal, respectively. On the other hand, at radial outflow locations, the extremes of $N u_{\mathrm{i}}$ and $N u_{\mathrm{o}}$ are locally minimal and locally maximal, respectively. Furthermore, as Fig. 4(a) shows, $N u_{\text {i }}$ and $N u_{\mathrm{o}}$ intersected at $\theta=57^{\circ}, 123^{\circ}$, which nearly corresponds to the centers of the two large-scale cells.

The isotherms for the 0 -vortex mode with pinches at $R e=713$ are shown in Fig. 3(b). The isotherms are almost parallel to the spherical boundaries everywhere, except at the stagnation points and radial outflow locations. Hence, the flow convection effect appeared in the neighborhood of the equator. Fig. 4(b) shows the local Nusselt number distributions for this mode. At the poles, $N u_{\mathrm{i}}$ was locally maximal, and decreased gradually as the fluid moved down from the poles along the inner sphere. As it approached the stagnation points, the fluid was heated by the hot outflow beside the stagnation points, where heat transfer and $N u_{\mathrm{i}}$ decreased significantly. In the small-scale cells, the 


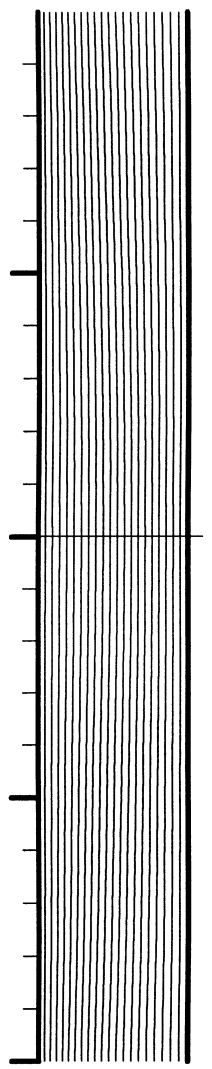

(a) $\operatorname{Re}=514$

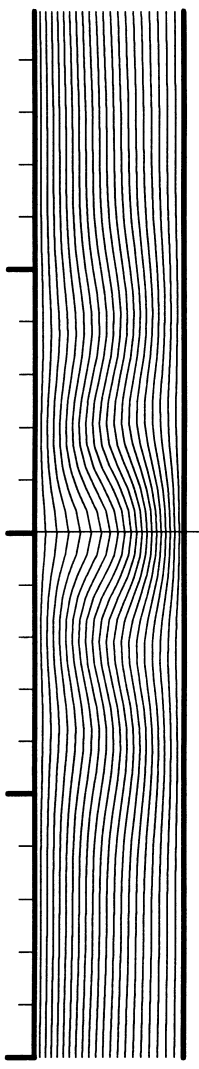

(b) $\operatorname{Re}=713$

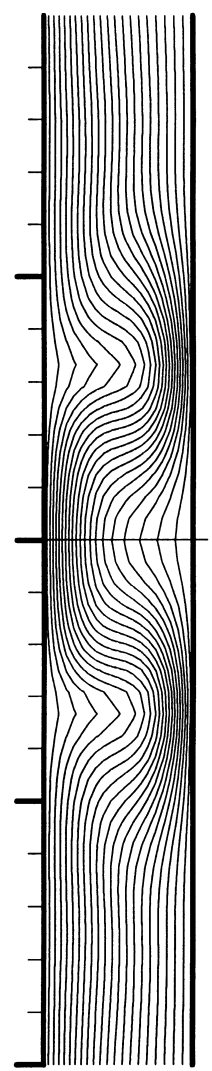

(c) $\operatorname{Re}=1212$

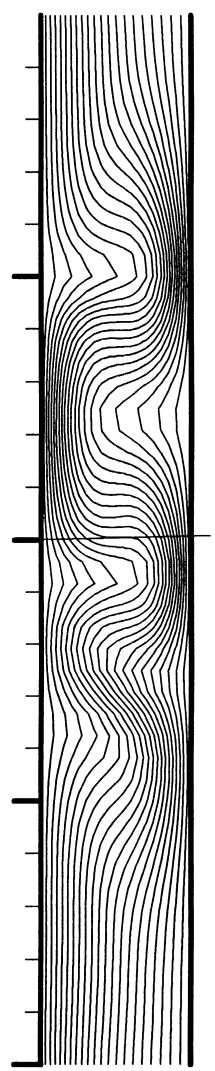

(d) $\mathrm{Re}=1178$

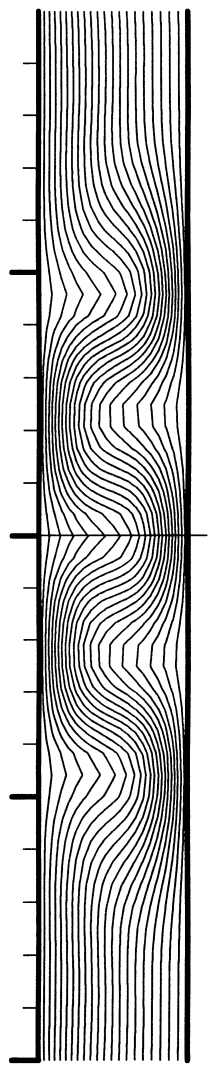

(e) $\operatorname{Re}=906$

\section{Indicates the position of the equator.}

Fig. 3. Isotherms of different flow modes for $\sigma=0.18$.

inner spheres suffered from the direct impact of the cold fluid in the outer sphere at the inflow boundaries beside the stagnation points. Therefore, local $N u_{\mathrm{i}}$ maximal and minimal formed at the outflow and inflow boundaries beside the stagnation points. Fluid moving to the equator along the inner sphere was then heated again by the hot wall and outflow at the equator. Meanwhile, $N u_{\mathrm{i}}$ decreased significantly again forming a local $N u_{\mathrm{i}}$ minimum at the equator. By contrast, at the equator, the outer sphere suffered from the impact of the hot radial outflow, and heat transfer was at its greatest, forming a locally maximal $N u_{\mathrm{o}}$ distribution. Fluid returning to the stagnation points along the outer sphere was then cooled by the cold wall, therefore, $N u_{\mathrm{o}}$ was locally minimal at the small-scale cell inflow boundaries. In the large-scale cells, the outer spheres suffered from the direct impact of the hot fluid in the inner spheres at the outflow boundaries beside the stagnation points forming a locally maximal $N u_{\mathrm{o}}$. Fluid returning to the poles was then cooled by the cold walls, and $N u_{\mathrm{o}}$ decreased gradually. Extremes of
$N u_{\mathrm{i}}$ and $N u_{\mathrm{o}}$ also existed at the inflow and outflow boundaries in this mode. Fig. 4(b) also shows that $N u_{\mathrm{i}}$ and $N u_{\mathrm{o}}$ intersected at $\theta=63^{\circ}, 117^{\circ}$ which nearly corresponds to the large-scale cell center locations.

Fig. 3(c) shows the isotherms of the 1-vortex mode. The streamlines distribution of the mode, as presented in Fig. 2(c), show radial inflow formed at the poles and the equator, and radial outflow formed between the vortices and large-scale cells on either side of the equator. Furthermore, isotherms twisted outward or inward at locations corresponding to the radial outflow and inflow. Fig. 4(c) shows the local Nusselt number distributions for this mode. $N u_{\mathrm{i}}$ and $N u_{\mathrm{o}}$ distribution tendencies can thus be determined by these radial inflow and outflow characteristics. At the poles and equator, $N u_{\mathrm{i}}$ and $N u_{\mathrm{o}}$ are locally maximal and minimal, respectively. However, at the boundaries between the vortices and large-scale cells, $N u_{\mathrm{i}}$ and $N u_{\mathrm{o}}$ are locally minimal and maximal, respectively. Furthermore, $N u_{\mathrm{i}}$ and $N u_{\mathrm{o}}$ intersected at $\theta=63^{\circ}, 83^{\circ}$ and $\theta=97^{\circ}, 117^{\circ}$, which nearly correspond to the lo- 
(a)

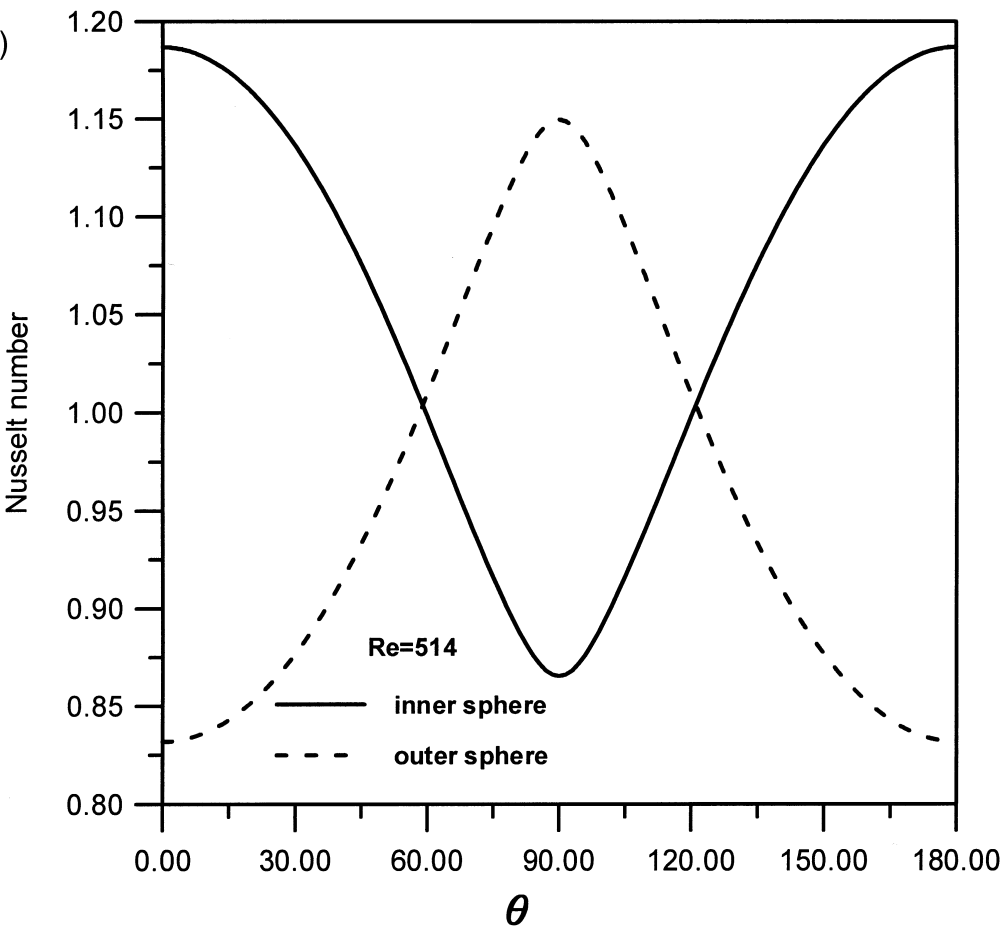

(b)

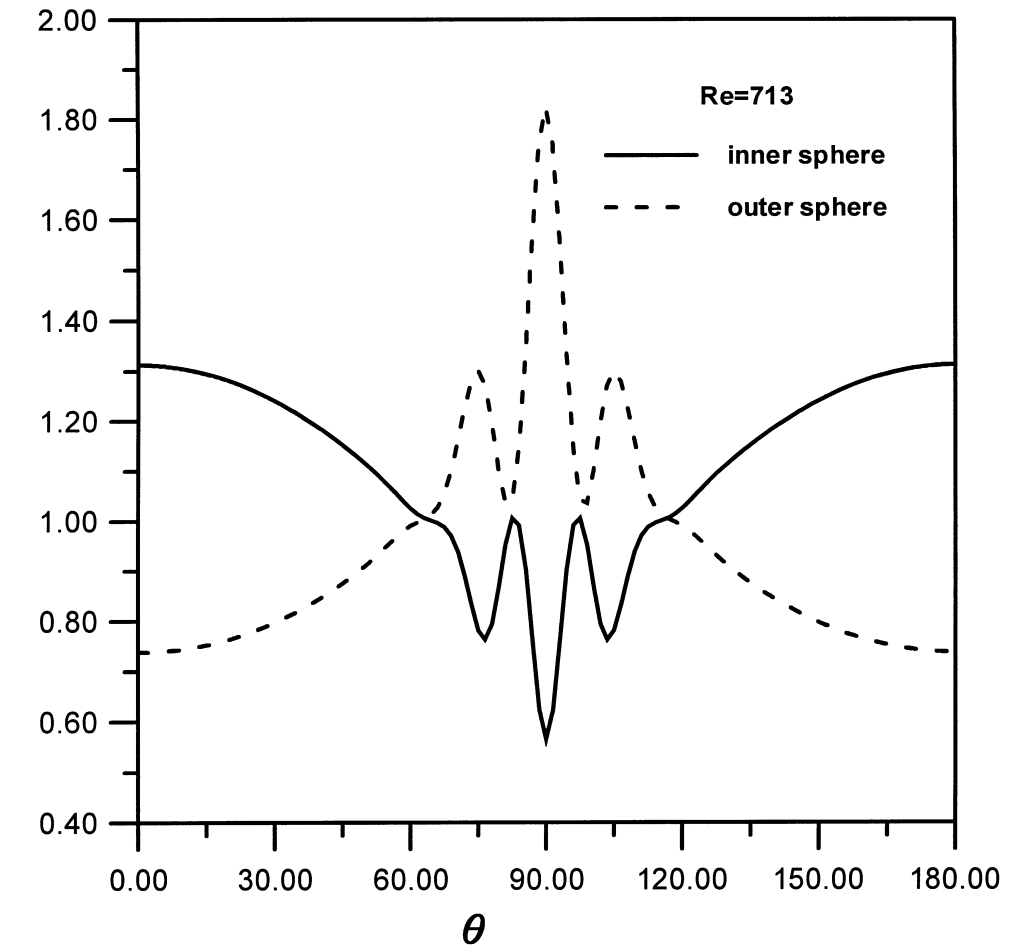

Fig. 4. (a) Local Nusselt number distribution of zero vortex mode. (b) Local Nusselt number distribution of zero vortex mode with pinches. (c) Local Nusselt number distribution of one vortex mode. (d) Local Nusselt number distribution of asymmetric one-vortex with a pinch mode. (e) Local Nusselt number distribution of two vortex mode. 

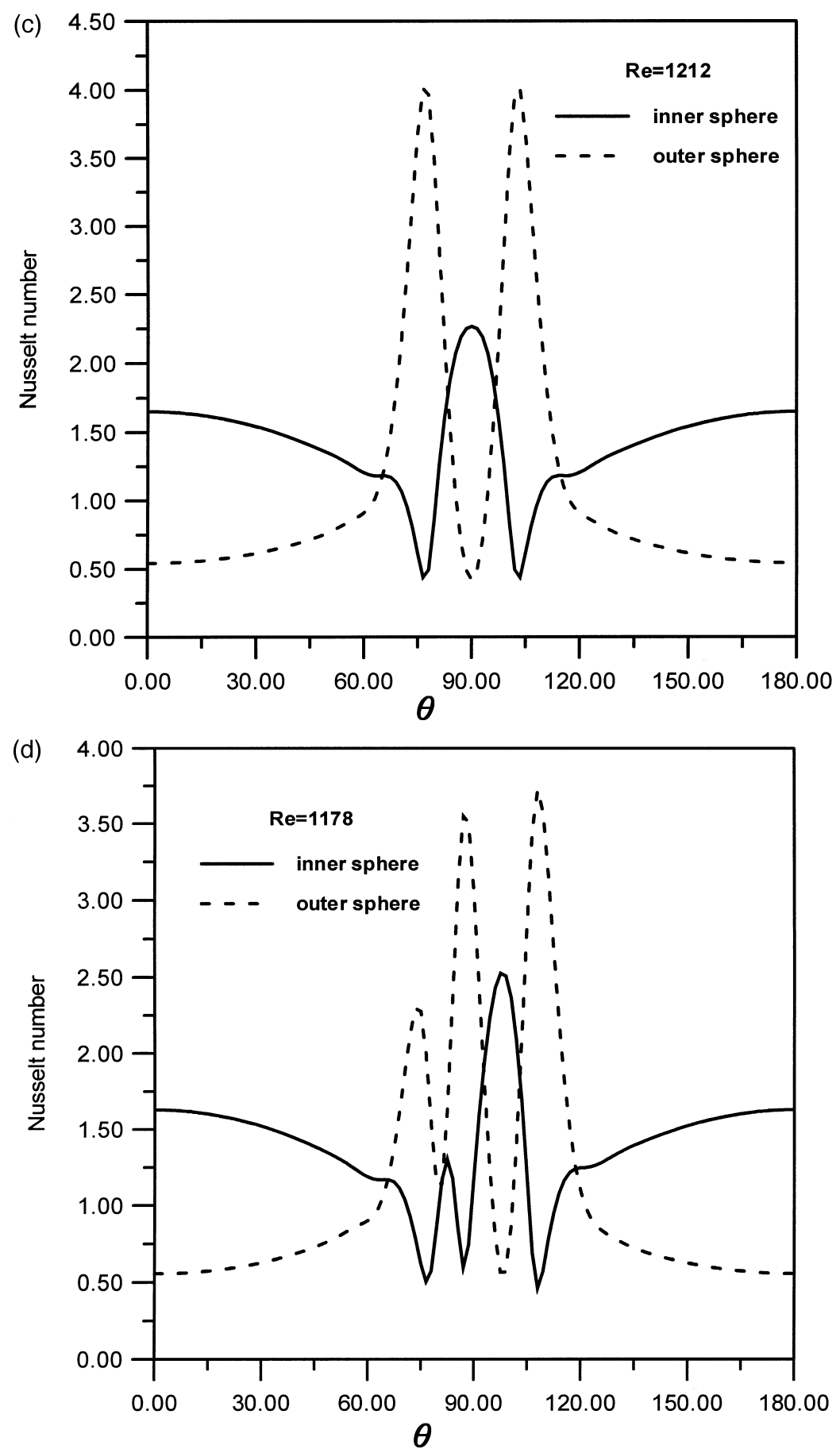

Fig. 4 (continued) 


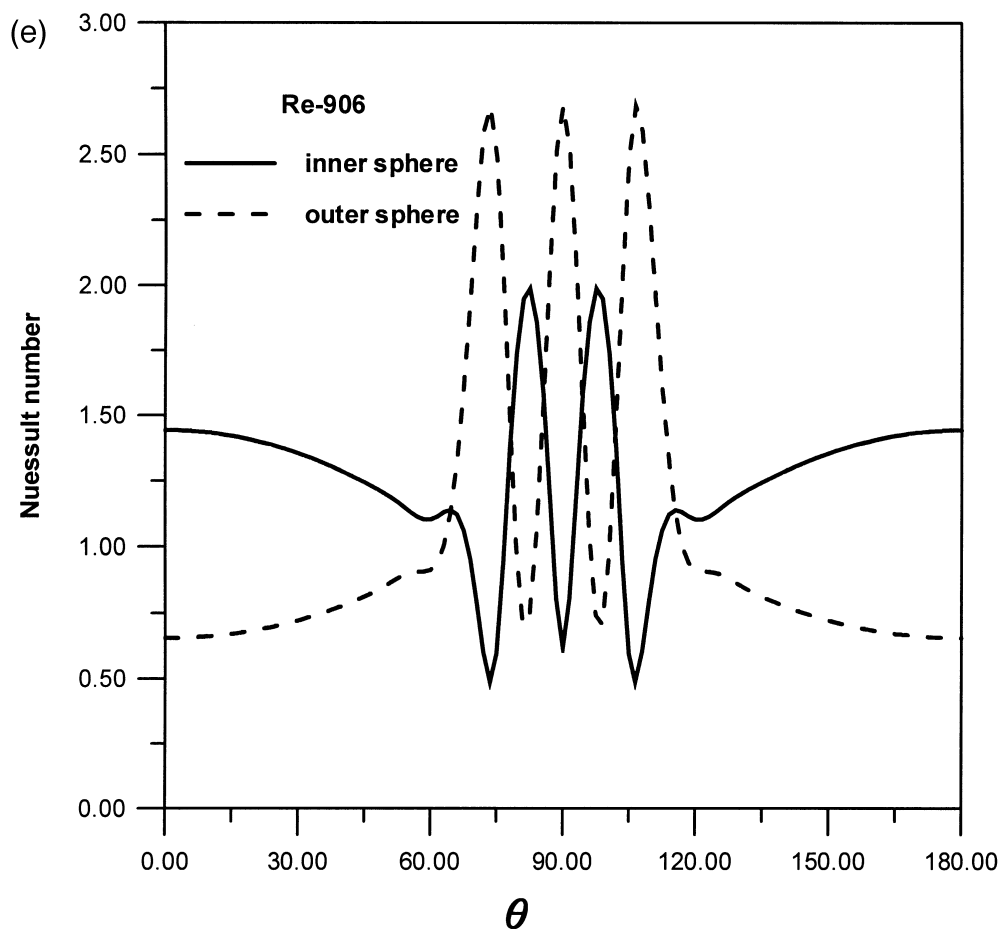

Fig. 4 (continued)

cations of the large-scale cell centers and vortices on either side of the equator.

The isotherms and the local Nusselt number distributions for the asymmetric 1-vortex with a pinch mode at $R e=1178$ are shown in Figs. 3(d) and 4(d), respectively. In the equatorial neighborhood, the isotherms twisted outward and inward at locations corresponding to the radial inflow and outflow. $N u_{\mathrm{i}}$ and $N u_{\mathrm{o}}$ distribution tendencies can also be determined by the inflow and outflow characteristics. Therefore, at the poles, between the vortices, and at the inflow locations beside the stagnation points, $N u_{\mathrm{i}}$ and $N u_{\mathrm{o}}$ are locally maximal and locally minimal, respectively. However, at the boundaries between the vortices and the large-scale cells on either side of the equator, and at the outflow locations beside the stagnation points, $N u_{\mathrm{i}}$ and $N u_{\mathrm{o}}$ are locally minimal and locally maximal, respectively. Figs. 3(d) and 4(d) also show that $N u_{\mathrm{i}}$ and $N u_{\mathrm{o}}$ intersected at $\theta=65^{\circ}, 92^{\circ}, 103^{\circ}$, and $119^{\circ}$, which nearly correspond to the large-scale cell center and vortex locations.

Figs. 3(e) and 4(e) show isotherms and the local Nusselt number distributions for the 2-vortex mode at $R e=906$. Isotherm distribution twisted outward or inward at the locations according to the respective outflow and inflow. Therefore, at the poles, and between the vortices on both sides of the equator, $N u_{\mathrm{i}}$ and $N u_{\mathrm{o}}$ were locally maximal and locally minimal, respectively. However, at the equator, and between the large-scale cells and vortices, $N u_{\mathrm{i}}$ and $N u_{\mathrm{o}}$ were locally minimal and maximal, respectively. $N u_{\mathrm{i}}$ and $N u_{\mathrm{o}}$ also intersected at $\theta=63^{\circ}, 83^{\circ}, \theta=63^{\circ}, 83^{\circ}$ and $\theta=63^{\circ}, 83^{\circ}$, which nearly correspond to the large-cell center and vortex locations.

In Figs. 5(a) and (b), Nusselt number distributions for stable 1-vortex and 2-vortex are shown at $R e=1000$. In Fig. 5(a), there are two peaks for 1 -vortex mode and three peaks for 2-vortex mode, respectively, corresponding to outflow boundaries on outer sphere. Due to reflection symmetric to the equator, the peak values of each mode are identical in northern and southern hemisphere. However, the peak values of 1vortex mode are $25 \%$ larger than that of 2 -vortex mode, and the minimum value of 1-vortex mode is still smaller than that of 2-vortex mode. At $R e=1000$, the 1 -vortex mode has the highest and lowest temperatures on outer sphere compared to those of the 2-vortex mode. On inner sphere, there are two peaks for 2-vortex mode and one peak for 1-vortex mode in Fig. 5(b). The peak values of 2-vortex mode are just 5\% larger than that of 1 -vortex mode, and the minimum value of 1 -vortex mode is also smaller than that of 2-vortex mode. In this case, the highest temperature occurs to the 1-vortex mode and the lowest temperature occurs 

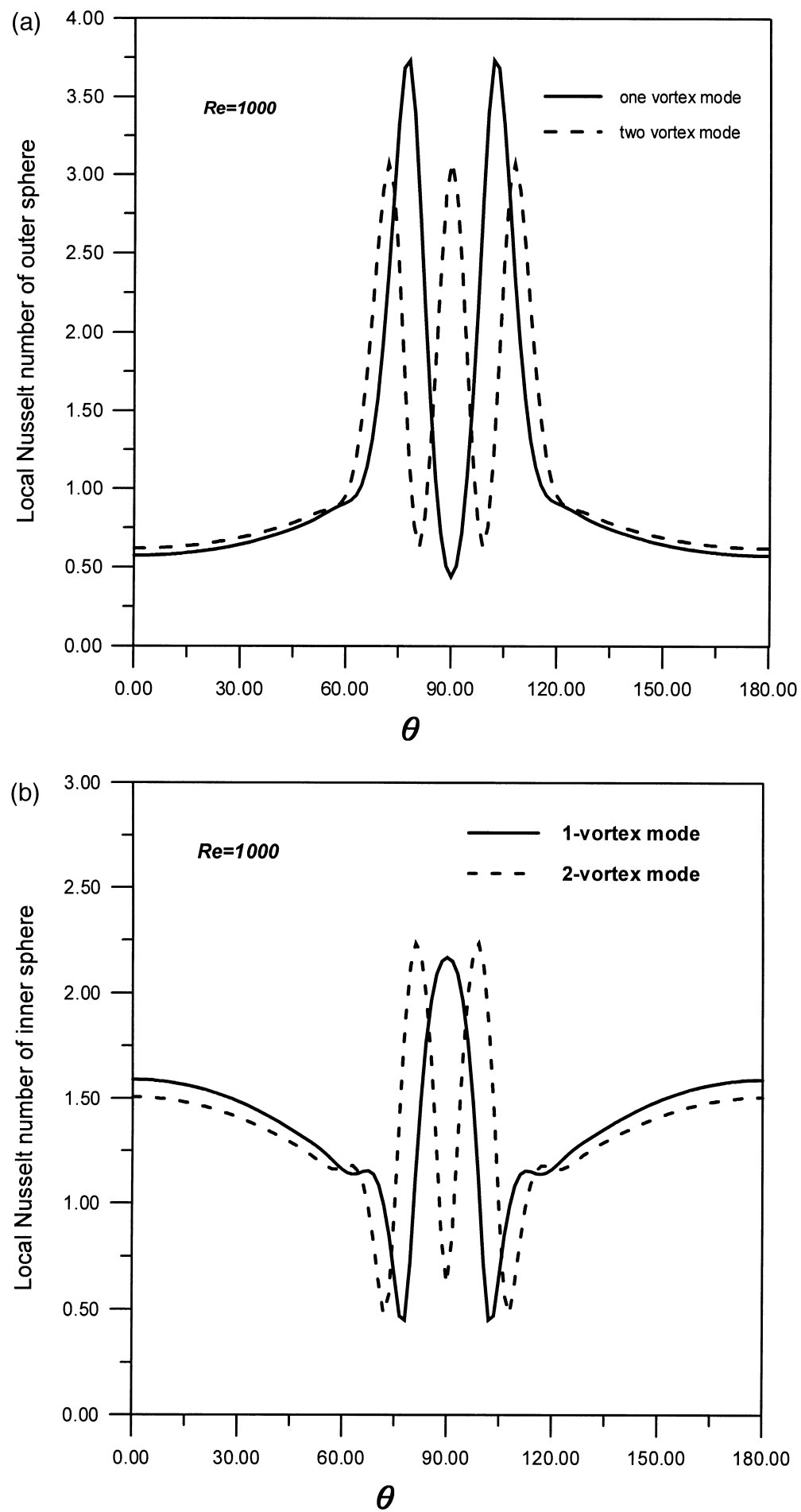

Fig. 5. (a) Local Nusselt number distribution of one-vortex and two-vortex modes on outer sphere at $R e=1000$. (b) Local Nusselt number distribution of one-vortex and two-vortex modes on inner sphere at $R e=1000$. 


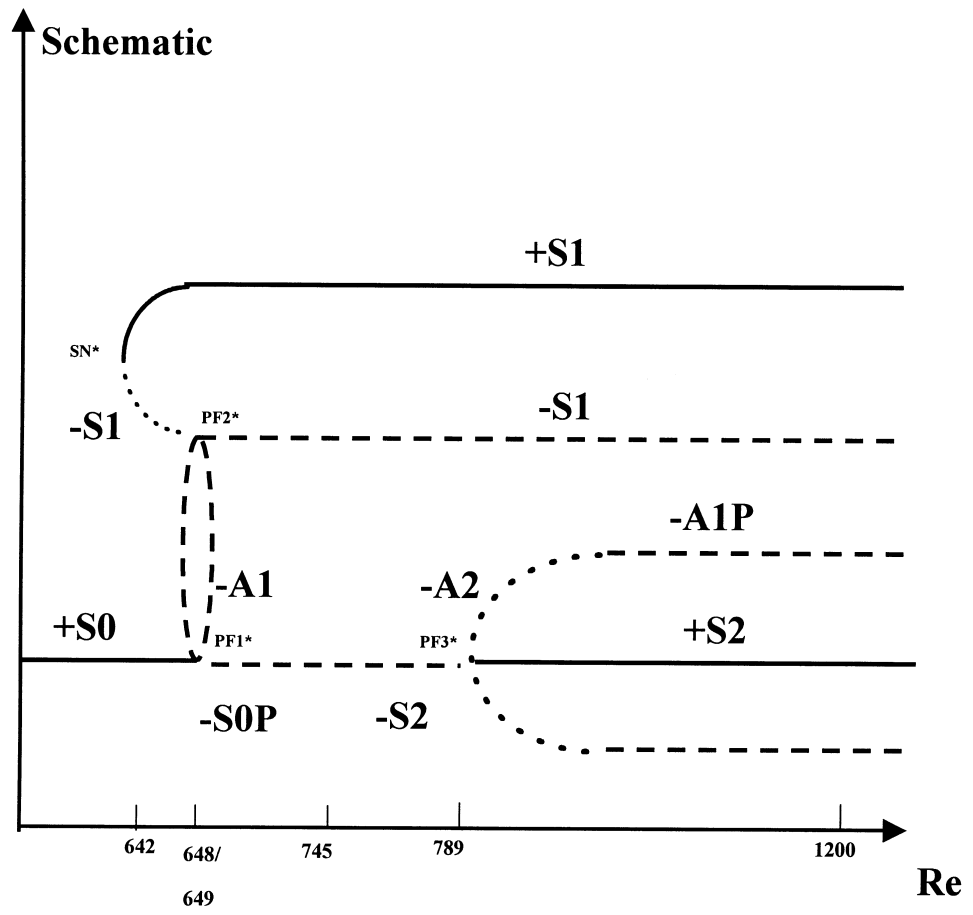

Fig. 6. Schematic bifurcation diagram.

to the 2-vortex mode, respectively. As can be shown later, the average Nusselt number for the 2-vortex mode is in general larger than that of the 1-vortex mode.

\subsection{Bifurcation diagram and average heat transfer}

A schematic representation of the bifurcation diagram is shown in Fig. 6. The vertical axis of Fig. 6 represents flow states and is not a monotonic-nor even a single-valued - function of torque. In the schematic bifurcation diagram for $R e<1200$, the stable (solid lines) and unstable (dash lines) branch will be labeled with the index of + and - , respectively. Linear stability analysis of the Jacobian matrix determines the character of the indices. The second index of the labels indicates whether the branch is symmetric or asymmetric. Therefore, a symmetric and an asymmetric branch will be labeled $\mathrm{S}$ and A, respectively. The third numeral index of the labels indicates the number of Taylor-vortex pairs in the flow. The fourth index $p$ indicates if the flow states possess pinches in its largescale recirculation or not.

We can see from Fig. 6 that the basic 0-vortex flow is stable $(+\mathrm{S} 0)$ at lower Reynolds numbers. Continuing the stable solution to higher Reynolds numbers, a subcritical pitchfork bifurcation point (PF1) was detected at $\mathrm{Re}=649$. Via this pitchfork bi- furcation, two solution branches are found, namely, an unstable 0 -vortex flow with pinches $(-\mathrm{S} 0 \mathrm{P})$ along the primary branch and an unstable symmetry-breaking transition branch $(-\mathrm{A} 1)$ to 1 -vortex flow. Along the $(-\mathrm{A} 1)$ branch, i.e. $0 \rightarrow 1$ vortex branch, a pitchfork bifurcation $(\mathrm{PF} 2)$ occurs at $R e=648$. After this bifurcation point, there are two solution branches:

1. Along (-S1) branch (left to the PF2), which has one positive eigenvalue through the linear stability analysis. This branch will be stabilized via a saddlenode bifurcation (SN) at $R e=642$ to the stable symmetric 1-vortex flow $(+\mathrm{S} 1)$,

2. Along (-S1) branch (right to the PF2), which has two positive eigenvalues and is called as twice unstable symmetric 1-vortex flow.

We now follow the stable 1-vortex branch $(+\mathrm{S} 1)$. With the increase in Reynolds number, the vortex strength along this solution branch grew stronger gradually.

We now follow the branch of 0 -vortex with pinches $(-\mathrm{S} 0 \mathrm{P})$. With the increase in $R e$, the flow gradually became an unstable 2-vortex flow. This unstable 2-vortex flow will undergo another subcritical pitchfork bifurcation (PF3) at $R e=789$, leading to a new unstable asymmetric 2-vortex flow (-A2). The asymmetry increases along its branch. However, along the parent branch, it passes through PF3, changing the index from $-\mathrm{S} 2$ to $+\mathrm{S} 2$, where the pitchfork bifur- 


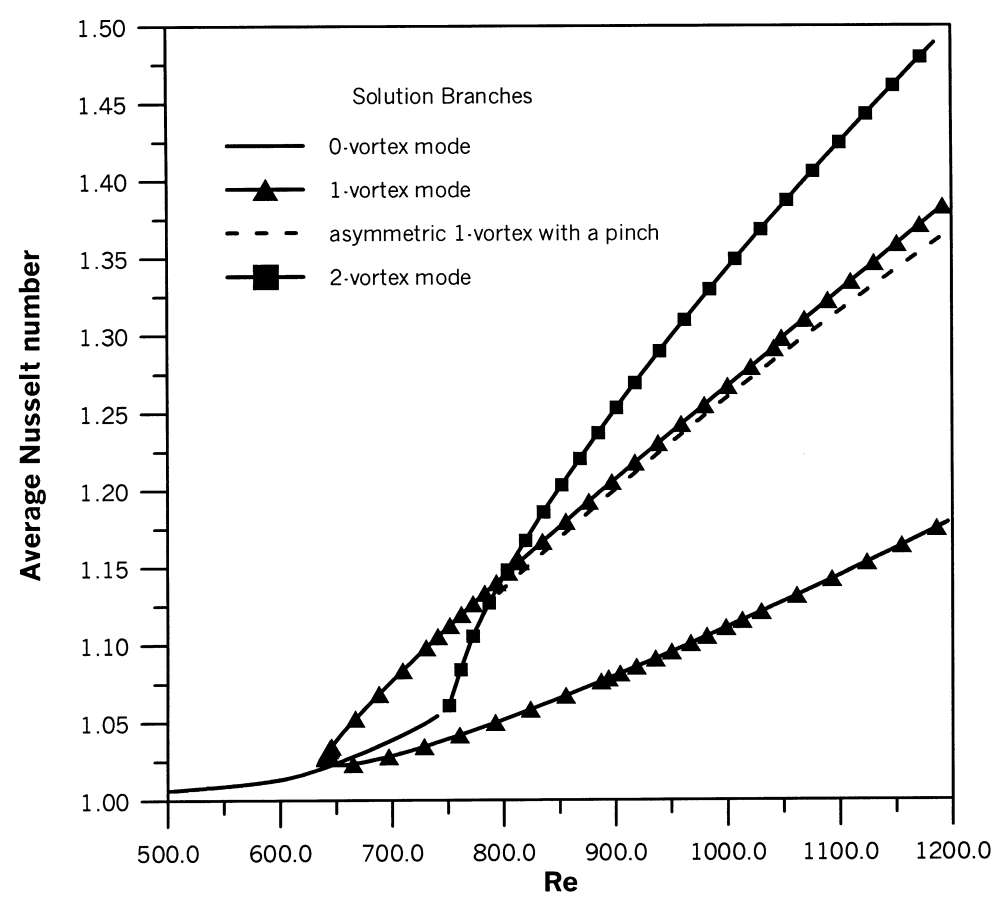

Fig. 7. Average Nusselt number for different modes at various Reynolds numbers.

cation restabilizes the parent branch. Along each unstable asymmetric two-vortex branch, each branch gradually transforms into another newly found unstable asymmetric 1-vortex with a pinch state.

Heat was transferred purely by conduction when the average Nusselt number $\left(\bar{N} u_{\mathrm{i}, 0}\right.$ as defined in Eq. (25)) was equal to one. When the average Nusselt number was greater than one, heat was transferred by conduction and also by flow convection. Fig. 7 show $\bar{N} u_{\mathrm{i}, 0}$ for different modes at various Reynolds numbers. In energy conversion between the inner and outer spheres, $\bar{N} u_{\mathrm{i}}$ must be equal to $\bar{N} u_{\mathrm{o}}$ for the arbitrary solution mode. Hence, only $\bar{N} u_{\mathrm{i}}$ distribution is represented in this figure.

We can see from Fig. 7 that at lower Reynolds numbers there was only stable 0 -vortex state $(+\mathrm{S} 0)$ existing, and the average Nusselt number was almost equal to one. Therefore, heat was transferred mainly by pure conduction in this solution mode. Via the pitchfork bifurcation (PF1) at $R e=649$, two solution branches are found, namely, an unstable 0-vortex flow with pinches $(-\mathrm{S} 0 \mathrm{P})$ along the primary branch and an unstable symmetry-breaking transition branch $(-\mathrm{A} 1)$ to 1 -vortex flow. We now follow the stable 1 -vortex branch $(+\mathrm{S} 1)$ and the unstable 1-vortex branch $(-\mathrm{S} 1)$. With the increase in Reynolds number, the vortex strength and flow convection effect along these solution branches grew stronger, and $\bar{N} u_{\mathrm{i}}$ of both branches increased gradually. Moreover, the dis- crepancy of $\bar{N} u_{\mathrm{i}}$ for these two branches was gradually on the increase with the increase in $R e$.

We now follow the branch of 0 -vortex with pinches (-S0P), where $\bar{N} u_{\mathrm{i}}$ rose slightly. With the increase in $R e$, the flow gradually became an unstable 2-vortex flow. The vortex and flow convection effects grew stronger, and $\bar{N} u_{\mathrm{i}}$ rose significantly with the increase in $R e$. Moreover, $\bar{N} u_{\mathrm{i}}$ of the two-vortex mode exceeded that of the one-vortex mode at $R e=801$. The intersection of these two branches was an average Nusselt number projection, not a bifurcation point. This unstable 2-vortex flow will undergo another subcritical pitchfork bifurcation (PF3) at $R e=789$, leading to a new unstable asymmetric 2-vortex flow $(-\mathrm{A} 2)$. The asymmetry increases along its branch. $\bar{N} u_{\mathrm{i}}$ distribution of the asymmetric two-vortex flow almost overlap with the 2-vortex mode at the range of $R e$ between 789 and 808. Beyond the bifurcation of PF3, the convection effect was weaker along these asymmetric solution branches than that in the stable 2-vortex mode, but was close to that of the 1 -vortex mode. Therefore, $\bar{N} u_{\mathrm{i}}$ distributions of the 1-vortex and asymmetric 1-vortex with a pinch mode nearly parallel each other.

\section{Conclusions}

This paper investigates the steady flow and heat transfer between two concentric spheres with the inner 
one rotating and the outer one stationary for the gap width of $\sigma=0.18$. A continuation method has been developed and applied to find possible solution branches for $R e<1200$. The method also uses a test function to predict and locate singularities. As demonstrated in this study, the test function approach can effectively locate singular points. A bifurcation is indicated by a zero quantity of the test function. The test function is continuous in a sufficiently large interval for the cases considered in this study. The computed solutions are carefully checked to be grid-independent. Our results show reasonably good agreement with other numerical solutions and experimental data. Past studies have found the 0-, 1-, and 2-vortex flow modes at $\sigma=0.18$. However, besides the three flow modes, the present study found some more steady state solutions, namely, (1) the unstable asymmetric 2-vortex state (-A2, via a pitchfork bifurcation PF3); (2) the unstable asymmetric 1-vortex with a pinch state (-A1P, followed the $-\mathrm{A} 2)$, (3) the twice unstable symmetric 1 -vortex state $(-\mathrm{S} 1$, via a pitchfork bifurcation PF2).

For local heat transfer, at the radial inflow locations, $N u_{\mathrm{i}}$ is locally maximal and $N u_{\mathrm{o}}$ is locally minimal, while at the radial outflow locations, $N u_{\mathrm{i}}$ is locally minimal, and $N u_{\mathrm{o}}$ is locally maximal. $N u_{\mathrm{i}}$ and $N u_{\mathrm{o}}$ intersect nearly at the centers of cells and vortices. For arbitrary Reynolds numbers between 808 and 1200, multiple solutions for the 1-vortex, 2-vortex, asymmetric 1-vortex with a pinch modes, and twice unstable 1-vortex mode exist simultaneously. Average heat transfer of the asymmetric 1-vortex with a pinch state and the 1-vortex state nearly parallel each other. Average heat transfer in the 2-vortex mode is larger than that in the 1-vortex mode, and that in the asymmetric 1-vortex with a pinch mode is smaller than 1vortex mode, and that in the twice unstable 1-vortex mode is the smallest of all.

\section{References}

[1] M. Wimmer, Experiments on a viscous fluid flow between concentric rotating spheres, J. Fluid Mech. 79 (1976) 317-335.

[2] F. Bartels, Taylor vortices between two concentric rotating spheres, J. Fluid Mech. 119 (1982) 1-25.

[3] P.S. Marcus, L.S. Tuckerman, Simulation of flow between concentric rotating spheres, Part 1 - steady states and Part 2 - transitions, J. Fluid Mech. 185 (1987) 1-65.

[4] G. Schrauf, The first instability in spherical
Taylor-Couette flow, J. Fluid Mech. 166 (1986) 287303.

[5] H.B. Keller, Numerical solution of bifurcation and nonlinear eigenvalue problems, in: P. Rabinowitz (Ed.), Applications of Bifurcation Theory, Academic Press, 1977, pp. 359-384.

[6] K. Bühler, Symmetric and asymmetric Taylor vortex flow in spherical gaps, Acta Mechanica 81 (1990) 3-38.

[7] P. Bar-Yoseph, A. Solan, R. Hillen, K.G. Roesner, Taylor vortex flow between eccentric coaxial rotating spheres, Phys. Fluids 2 (9) (1990) 1564-1573.

[8] C.K. Mamun, L.S. Tuckerman, Asymmetry and Hopf bifurcation in spherical Couette flow, Phys. Fluids 7 (1) (1995) 80-91.

[9] R.-J. Yang, A numerical procedure for predicting multiple solutions of a spherical Taylor-Couette flow, International Journal for Numerical Methods in Fluids 22 (1996) 1135-1147.

[10] E.H. Bishop, R.S. Kolflat, L.R. Mack, J.A. Scanlan, Convective heat transfer between concentric spheres, in: W.H. Giedt, S. Levy (Eds.), Proceedings of 1964 Heat Transfer Fluid Mech. Inst., Stanford University Press, Berkeley, CA, 1964, pp. 69-80.

[11] E.H. Bishop, J.A. Scanlan, L.R. Mack, Heat transfer by natural convection between concentric spheres, Int. J. Heat Mass Transfer 9 (1966) 649-662.

[12] J.A. Scanlan, E.H. Bishop, R.E. Powe, Natural convection heat transfer between concentric spheres, Int. J. Heat Mass Transfer 13 (1970) 1857-1872.

[13] S.H. Yin, R.E. Powe, J.A. Scanlan, E.H. Bishop, Natural convection flow patterns in spherical annuli, Int. J. Heat Mass Transfer 16 (1973) 1785-1795.

[14] P.J. Burns, C.L. Tien, Natural convection in porous media bounded by concentric spheres and horizontal cylinders, Int. J. Heat Mass Transfer 22 (1979) 929-939.

[15] S.N. Singh, J.M. Elliott, Natural convection between concentric spheres in a slightly-thermally stratified medium, Int. J. Heat Mass Transfer 24 (1981) 395-406.

[16] J.M. Nelsen, R.W. Douglass, On partial spectral expansions with natural convection in spherical annulus enclosures as an example, Numer. Heat Transfer 6 (1983) 67-84.

[17] J.M. Nelsen, R.W. Douglass, Non-uniform energy generation effects on natural convection in a spherical annulus enclosure, Int. J. Heat Mass Transfer 27 (1984) 1925-1928.

[18] J.L. Wright, R.W. Douglass, Natural convection in narrow-gap, spherical annuli, Int. J. Heat Mass Transfer 29 (1986) 725-739.

[19] R. Seydel, From Equilibrium to Chaos: Practical Bifurcation and Stability Analysis, Elsevier, New York, 1988.

[20] Y. Saad, Numerical Methods for Large Eigenvalues Problems, Halsted, 1992.

[21] D.C. Sorensen, Implicit application of polynomial filters in a k-step Arnoldi method, SIAM J. Matrix Anal. Appl. 13 (1992) 357-367. 